\title{
Sound radiation from railway sleepers
}

\author{
Xianying Zhang, David J Thompson, Giacomo Squicciarini
}

Institute of Sound and Vibration Research, University of Southampton, Southampton SO17 1BJ, UK

E-mail address: $\underline{x z 24 g 12 @ \text { soton.ac.uk }}$

\begin{abstract}
The sleepers supporting the rails of a railway track are an important source of noise at low frequencies. The sound radiation from the sleepers has been calculated using a threedimensional boundary element model including the effect of both reflective and partially absorptive ground. When the sleeper flexibility and support stiffness are taken into account, it is found that the radiation ratio of the sleeper can be approximated by that of a rigid half-sleeper. When multiple sleepers are excited through the rail, their sound radiation is increased. This effect has been calculated for cases where the sleeper is embedded in a rigid or partially absorptive ground. It is shown that it is sufficient to consider only three sleepers in determining their radiation ratio when installed in track. At low frequencies the vibration of the track is localised to the three sleepers nearest the excitation point whereas at higher frequencies the distance between the sleepers is large enough for them to be treated independently. Consequently the sound radiation increases by up to $5 \mathrm{~dB}$ below $100 \mathrm{~Hz}$ compared with the result for a single sleeper whereas above $300 \mathrm{~Hz}$ the result can be approximated by that for a single sleeper. Measurements on a $1 / 5$ scale model railway track are used to verify the numerical predictions with good agreement being found for all configurations.
\end{abstract}

\section{Introduction}

Conventional railway track consists of steel rails attached via rail pads and clips to transverse sleepers (cross ties) which are supported in ballast. In modern track the sleepers are usually made of pre-stressed concrete although timber and steel are also in use. In most situations the dominant source of noise from the railway system for conventional speeds is rolling noise. This is radiated by the wheels, the rails and, at low frequencies, also the sleepers.

In recent years numerical models have been developed that can be used to predict rolling noise and to separate the sound radiated by the various components. The first models of rolling noise were developed in the 1970s by Remington [1, 2]. Analytical models were used for the wheel 
and rail impedances and for their radiation efficiencies but the sound radiation of the sleepers was not considered. Nevertheless, Remington's work took account of many features that are still considered to be important: the relative displacement excitation by the wheel/rail roughness, the contact filter effect, track decay rates, etc. Subsequently, Thompson [3] extended this basic model to include other features that were found to be significant. This was implemented in the TWINS (Track-Wheel Interaction Noise Software) package [3] which was validated through field tests $[4,5]$. It has been found the sleeper radiation is the dominant component at low frequency, the rail radiation is important between 400 and $2000 \mathrm{~Hz}$ and the wheel radiation is significant at high frequency. Despite its widespread use, there remain areas for improvement in this model, in particular at low frequencies (below $300 \mathrm{~Hz}$ ) where the agreement with measurements is often less satisfactory. In this region it is found that the sleepers radiate most of the noise.

The sleeper acoustic power is currently predicted in TWINS by adopting a simplified formulation for its radiation efficiency [6]. Radiation efficiency is the sound power normalised by the surface area, mean square velocity and acoustic impedance of air. The model in TWINS [6] is based on an approximate relation for the radiation from a rectangular piston set in an infinite baffle. However, this is modified at low frequencies to allow for the fact that multiple sleepers are close together compared with the acoustic wavelength and therefore can be considered to form a single composite source. The increase in the radiation efficiency compared with that of a single sleeper is estimated using a heuristic approach [6]. One aim of the present work is to provide a more rigorous estimate of this effect. In addition to the sound power, to predict the sound pressure at a receiver location, information is required about the directivity. In TWINS [6] this is assumed to be omnidirectional as the source is acting as a monopole.

The sleeper radiation has been explored by some authors aiming at reducing track noise. Vincent et al. [7] used TWINS to investigate the various parameters that affect the track noise. They concluded that the sound power radiated by the sleepers could be minimized by reducing the rail pad stiffness. However, this was shown to lead to an increase in the rail noise component. Other possible approaches to reduce the sleeper noise that were identified were to increase the sleeper mass or to reduce the area of its upper side.

Acoustic optimization of railway sleepers was investigated numerically by Nielsen [8]. He used a finite element model of the sleeper to determine its vibration due to a passing train and 
combined this with the radiation efficiency for a single sleeper obtained using a threedimensional boundary element model of a sleeper on a rigid plane. He studied the effect of changing the sleeper geometry on the radiated noise, concluding that a bi-block sleeper with appropriate dimensions and sufficient mass could be 2-3 $\mathrm{dB}(\mathrm{A})$ quieter than the reference monobloc design. Based on these results a modified sleeper design was tested within the Silent Track project $[9,10]$ in conjunction with a modified rail section. Together these gave a noise reduction of about $3 \mathrm{~dB}$ but the effect of the sleeper design was not quantified separately.

Some initial results for the sound radiation of a single sleeper as well as multiple sleepers, embedded in a rigid ground, are presented in [10]. These were obtained using the Rayleigh integral approach [11], which is based on the assumption that the vibrating surface is flush with a rigid ground. Apart from the above references, however, very little work has been carried out into the acoustic characteristics of the sleepers.

Attenborough et al. [12] considered the effect of replacing acoustically hard sleepers by porous concrete sleepers. They used boundary element calculations to show that the rail noise could be reduced by $1.5 \mathrm{~dB}(\mathrm{~A})$ by such porous concrete sleepers. However, this work focused on the absorptive properties of the sleepers and did not take account of the sound produced by their vibration. In [13] it has been shown, using a boundary element approach, that the ground impedance can have a significant effect on the radiation from the rail. Similar effects may be expected for the radiation from the sleeper itself and will be considered here.

In this paper, calculations of the sound radiation from a single sleeper obtained by using the boundary element method in three dimensions (3D) are first presented in Section 2. Results are presented in terms of radiation efficiency; no consideration is given to the directivity. Initially, the sleeper is considered to be located in free space. The ground is then introduced, first as a rigid reflecting surface and then as a partially absorptive surface, represented by its impedance, and the effects on the sound radiation are investigated. The fact that multiple sleepers are connected by the rails can lead to a change in their radiation efficiency [10]. This effect is explored in Section 3 for sleepers in close proximity to both a rigid and an absorptive ground. Finally, in Section 4, measurements on a scale model are presented to verify the numerical predictions. The work concentrates on concrete monobloc sleepers which are the most common form used in modern ballasted track. 


\section{Sound radiation of a single sleeper}

\subsection{Vibration of a single sleeper}

Before considering the sound radiation from a sleeper its vibrational behaviour is considered first. In track vibration models the rail is often represented as a beam and the sleepers by a layer of independent rigid bodies (masses) [14]. However, such an approach neglects the bending modes that monobloc sleepers exhibit in the frequency range of interest. A simple Timoshenko beam for a freely suspended sleeper was proposed by Grassie [15] who showed that it was sufficient to represent the sleeper as an equivalent uniform beam. This beam model was extended in $[6,10]$ to include the stiffness and damping of the ballast below the sleeper. In the model presented in [10], the sleeper was represented by a finite length, uniform Timoshenko beam with free ends and the ballast by a continuous elastic layer, the stiffness of which may be frequency-dependent. The forced response was studied using a wave approach. Results obtained using this approach are presented here.

Monobloc concrete sleepers usually have a cross-section similar to that shown in Figure 1 and a typical length of about $2.5 \mathrm{~m}$. Often they are tapered slightly towards the middle but, for simplicity, this is neglected here and a constant cross-section as in Figure 1 is adopted. This will also be convenient for comparison with experimental results in Section 4, where a 1/5 scale model of a sleeper with this cross-section has been used. The parameters used for this sleeper are listed in Table 1.

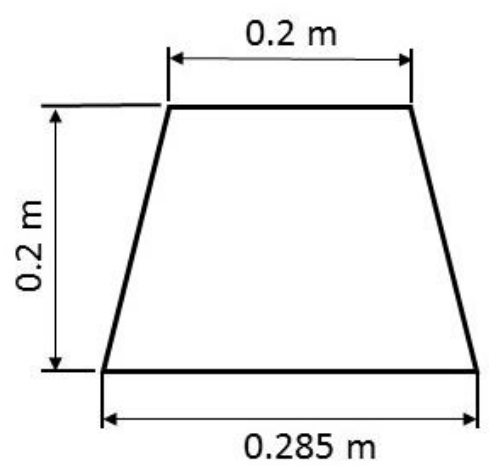

Figure 1. Cross-section of the idealised concrete sleeper 
Table 1 Parameters used to describe a concrete sleeper

\begin{tabular}{|c|c|}
\hline Young's modulus $\left(\mathrm{N} / \mathrm{m}^{2}\right)$ & $4.3 \times 10^{10}$ \\
\hline Shear modulus $\left(\mathrm{N} / \mathrm{m}^{2}\right)$ & $1.87 \times 10^{10}$ \\
\hline Poisson's ratio & 0.15 \\
\hline Sleeper damping loss factor & 0.01 \\
\hline Density $\left(\mathrm{kg} / \mathrm{m}^{3}\right)$ & 2500 \\
\hline Shear coefficient & 0.83 \\
\hline Length, $\mathrm{m}$ & 2.5 \\
\hline Excitation point, from one end $(\mathrm{m})$ & 0.5 \\
\hline Ballast damping loss factor & 1.0 \\
\hline
\end{tabular}

Figure 2 shows the calculated point mobility of the sleeper when it is excited at the rail seat. For the free sleeper a series of bending resonances can be seen; the first four modes have natural frequencies of 130, 350, 660 and $1040 \mathrm{~Hz}$. The amplitude of the first (and sixth) mode is much smaller than the adjacent modes as the rail seat is close to a nodal point. The displaced shape (operational deflection shape) at the first two of these frequencies is shown in Figure 3. This shows the real part of the response after normalisation to that at the excitation point $(y=0)$.

Figure 2 also shows the point mobility obtained in the presence of a frequency-dependent support stiffness, representing the ballast. This has a stiffness per unit length of $48 \mathrm{MN} / \mathrm{m}^{2}$ up to $100 \mathrm{~Hz}$, above which it increases linearly as shown in Figure 4(a) [10]. The damping is given by a constant loss factor of 1.0. The fundamental resonance of the beam on the foundation stiffness can be seen at about $100 \mathrm{~Hz}$. Above this frequency the bending resonances can still be seen but with reduced amplitude and increased natural frequency compared with the free beam. Figure 4(b) shows the spatially averaged mobility; this is the square root of the mean square mobility, which is of direct relevance to the radiated noise. 

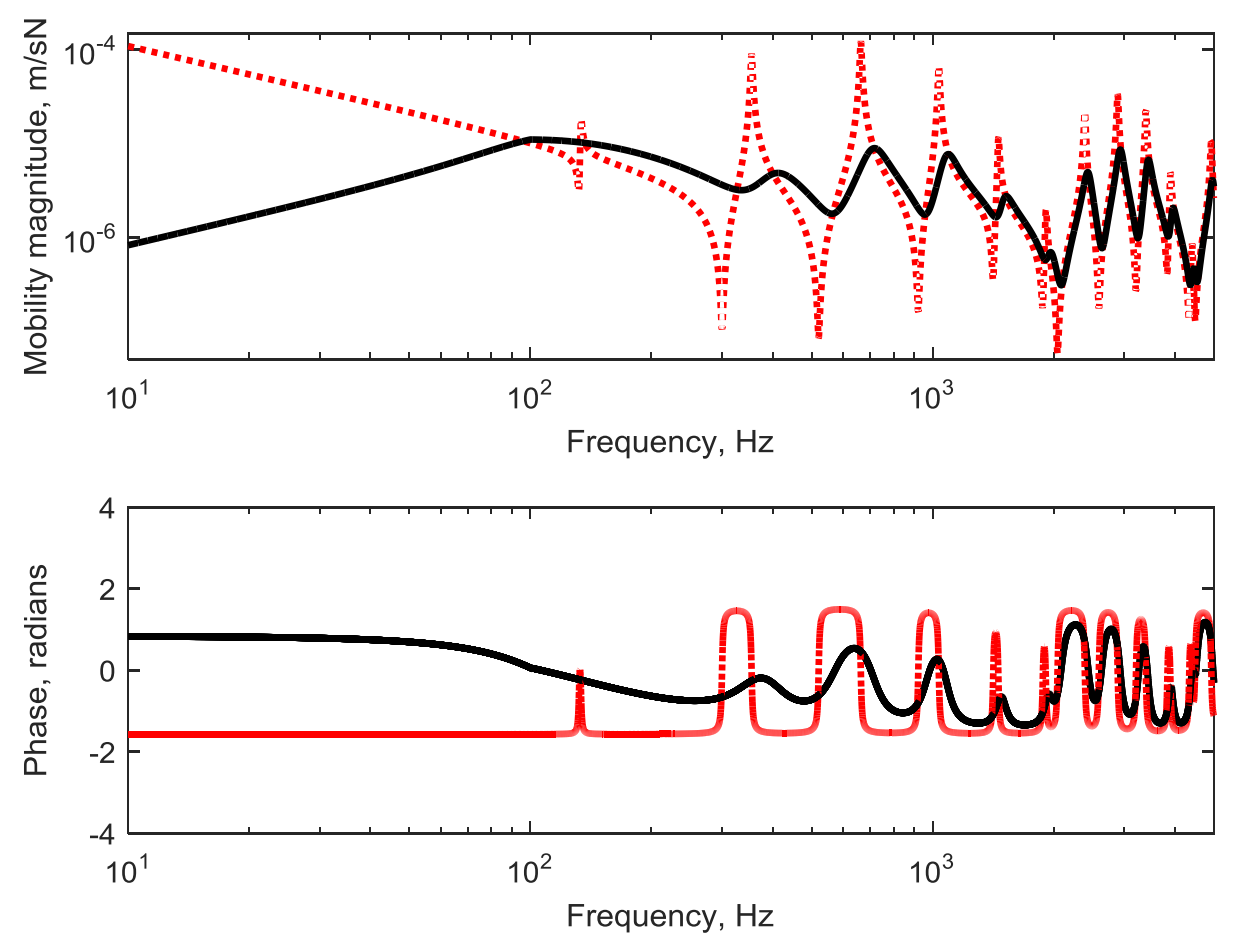

Figure 2. Point mobility of the concrete sleeper: $\cdots$, freely suspended sleeper; -, supported sleeper with frequency-dependent stiffness

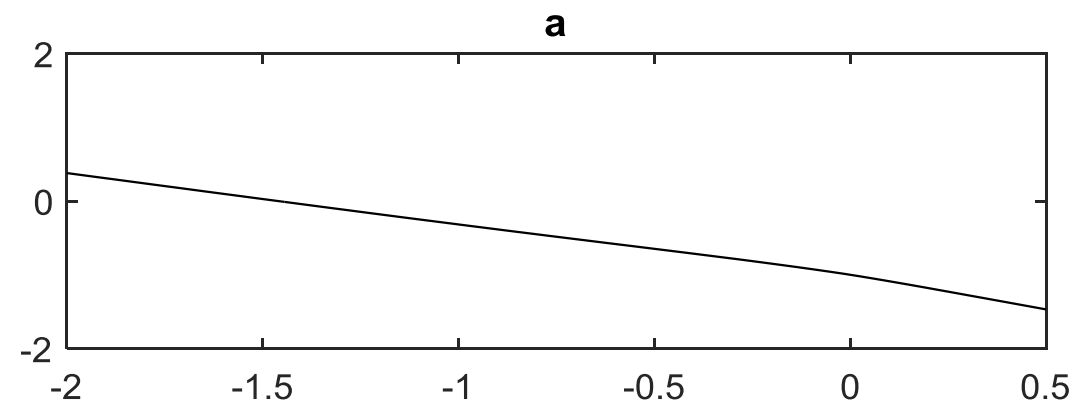

b



Figure 3. Displaced shape versus distance y along sleeper at frequencies corresponding to peaks of the mobility, normalised to response at excitation point. (a) $130 \mathrm{~Hz}$, (b) $350 \mathrm{~Hz}$. 

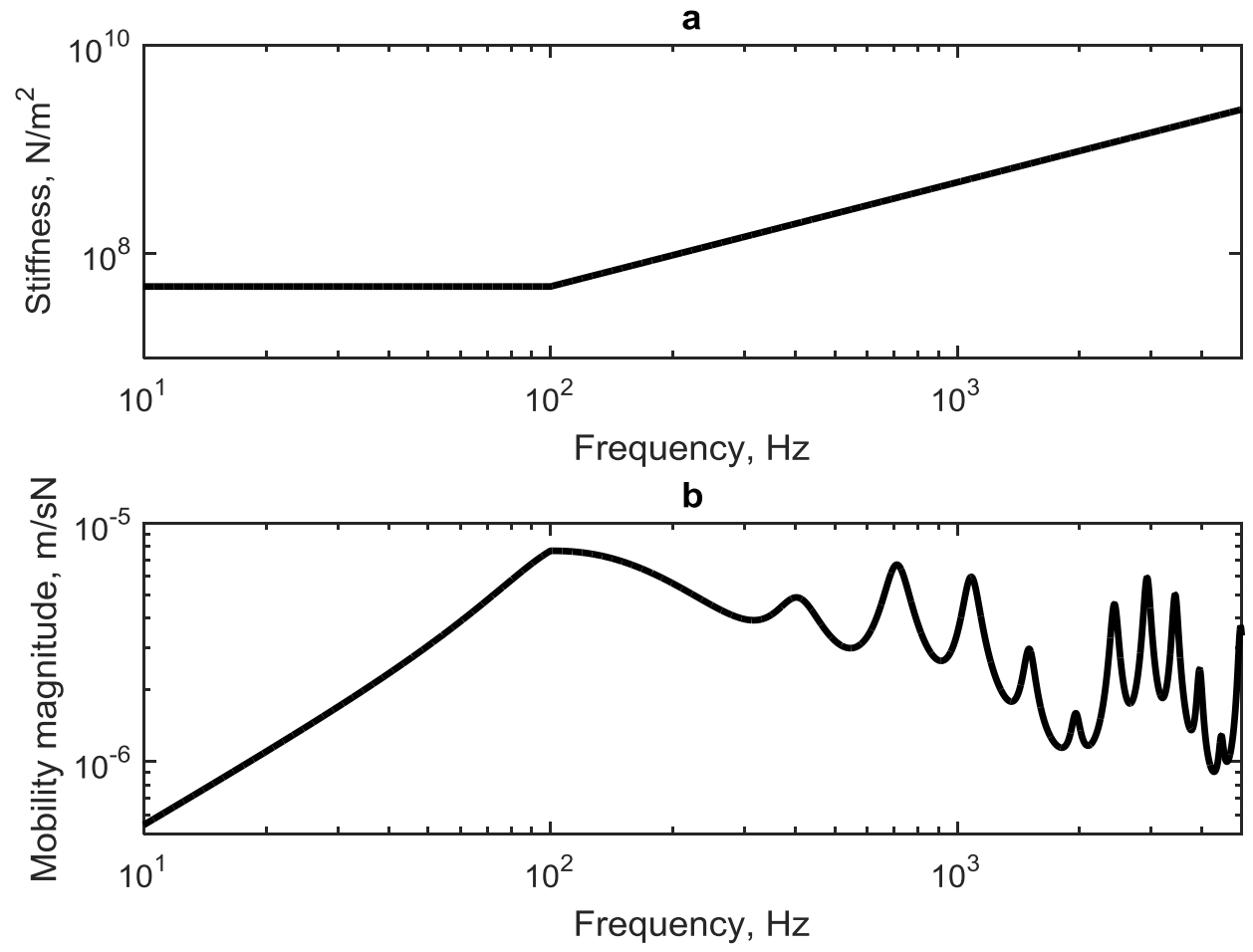

Figure 4. (a) Frequency-dependent ballast stiffness (per unit length). (b) Spatially-averaged mobility of sleeper with this ballast stiffness

\subsection{Radiation in free space}

To estimate the sound power radiated by a vibrating structure it is useful to define the radiation efficiency, or radiation ratio. It indicates the sound power $W$ a structure radiates compared with that from an ideal case of a baffled piston having the same surface area and vibrating with a mean-square velocity equal to the spatially-averaged mean-square velocity of the structure. This can be written as [16]

$$
\sigma=\frac{W}{\rho_{0} c_{0} S\left\langle v^{2}\right\rangle}
$$

where $\rho_{0}, c_{0}$ are the density of the air and the speed of sound, respectively, $W$ is the sound power radiated by the structure, $S$ is the total surface area and $\left\langle v^{2}\right\rangle$ is the surface-averaged mean-square normal velocity. The quantities $\sigma, W$ and $v$ are functions of frequency.

The sound radiation of a sleeper is modelled here using a three-dimensional boundary element model based on the direct BEM approach [17] and written in Matlab. For simplicity, the bending modes of the sleeper are ignored initially and a rigid body motion is assumed; the 
bending of the sleeper will be introduced in Section 2.3. A sleeper with the geometry given in Figure 1 is modelled first in free field by using 4-node quadrilateral boundary elements. Taking symmetry in two planes into account, the corresponding mesh for one quarter of the sleeper is shown in Figure 5. The planes shown are the corresponding symmetry planes in the model. The normal velocity is set equal to the component of the rigid body motion normal to the surface at each node when the sleeper vibrates vertically with unit amplitude.

Figure 6 presents the radiation ratio obtained. This is compared with the results for an infinitely long sleeper obtained using a two-dimensional (2D) boundary element model of the crosssection. The result from the 2D model has a slope of $f^{3}$ at low frequency (below $400 \mathrm{~Hz}$ ) and tends to unity at high frequency. This behaviour corresponds to that of an oscillating cylinder of radius approximately $0.15 \mathrm{~m}$ and may be considered as a line dipole source.

It can be seen there is a clear difference between the 2D and 3D predictions below about $140 \mathrm{~Hz}$, whereas at higher frequency their results are identical. From this it can be seen that the length of the sleeper is important at low frequencies, where the acoustic wavelength is large compared with the length (the acoustic wavelength is equal to $2.5 \mathrm{~m}$ at $136 \mathrm{~Hz}$ ). The slope of the curve below $100 \mathrm{~Hz}$ is $f^{4}$, corresponding to a point dipole. It follows the slope of $f^{3}$ in the intermediate frequency region between about 140 and $350 \mathrm{~Hz}$ before tending to unity as for the 2D model.



Figure 5. Mesh of one quarter of the sleeper

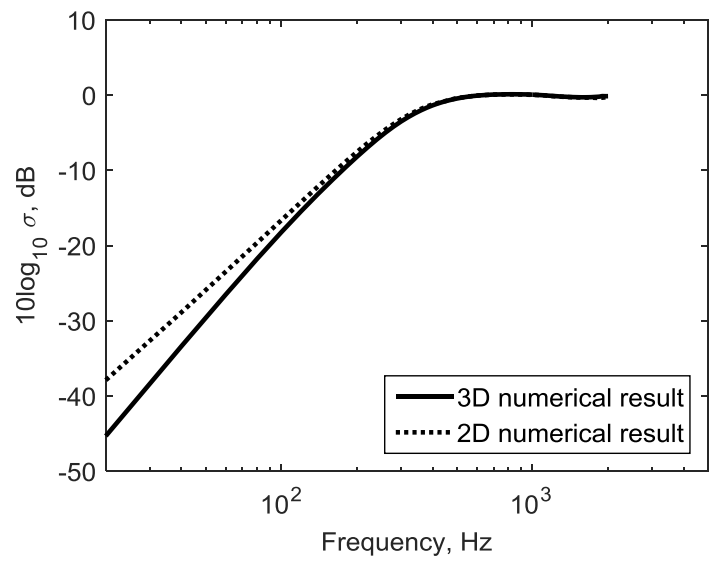

Figure 6. Sound radiation ratio of a $2.5 \mathrm{~m}$ long sleeper in free space 


\subsection{Influence of a rigid ground on the sleeper radiation}

The sleeper in free space is not a good representation of the actual situation; instead it is usually located on the ground in ballast. It is necessary, therefore, to investigate the effects of the presence of the ground on the sleeper radiation.

For simplicity, the ground is assumed initially to be rigid. Only the upper surface of the sleeper is assumed to be visible, the remainder being fully embedded in the ground. It is assumed in this situation that only the top surface of the sleeper radiates sound, this being considered flush with the ground surface. The rigid ground can be represented in the boundary element model by an infinite reflecting (symmetry) plane [17]. There are now therefore three orthogonal symmetry planes in the model, with the rectangular upper surface of the sleeper represented by boundary elements. Figure 7 shows the radiation ratio of the sleeper in this case. Also, in this idealised case, the Rayleigh integral method [11] could be used instead of the boundary element method. It has been verified that the numerical results from these two approaches agree very well with each other (the Rayleigh integral method is used for convenience in Section 3.2 below).

The radiation ratio has a slope of $f^{2}$ at low frequency (below $70 \mathrm{~Hz}$ ), corresponding to the behaviour of a point monopole. In this region the acoustic wavelength is larger than the sleeper length. Between about $80 \mathrm{~Hz}$ and $600 \mathrm{~Hz}$, half the acoustic wavelength is smaller than the sleeper length but greater than its width, and the sleeper has the characteristic of a line monopole with a slope proportional to $f$. Above $800 \mathrm{~Hz}$, the radiation ratio again tends to unity. If the sleeper length is changed to $1.25 \mathrm{~m}$, it can be seen that the radiation ratio is reduced by $3 \mathrm{~dB}$ below about $100 \mathrm{~Hz}$ but is unaffected above $200 \mathrm{~Hz}$.

It is also possible to include the effects of the sleeper flexibility in this model. The beam model described in Section 2.1 is used to determine the vertical vibration amplitude at each position along the sleeper corresponding to the nodes of the BEM mesh. The force point is again considered to be at the rail seat. On each cross-section the normal velocity is determined from this vertical amplitude in the same way as before. The results are shown in Figure 7 together with those for the case with rigid body motion. As can be seen, the flexible motion of the sleeper causes it to radiate less noise below about $400 \mathrm{~Hz}$, whereas the results are the same at higher frequency. Below $100 \mathrm{~Hz}$ the sleeper vibration consists of a combination of vertical translation and rotation, as shown in Figure 3, with the centre of rotation close to the position of the other rail. There is therefore some cancellation in the radiation from that end of the 
sleeper. Consequently it is found that the result for the flexible sleeper corresponds to that for a shorter rigid sleeper; in fact it is close to that for a rigid sleeper of length $1.25 \mathrm{~m}$. This therefore gives a better approximation of the behaviour of the flexible sleeper than the full length model. Therefore, in the remainder of this paper the rigid half-sleeper of length $1.25 \mathrm{~m}$ will be used for simplicity.

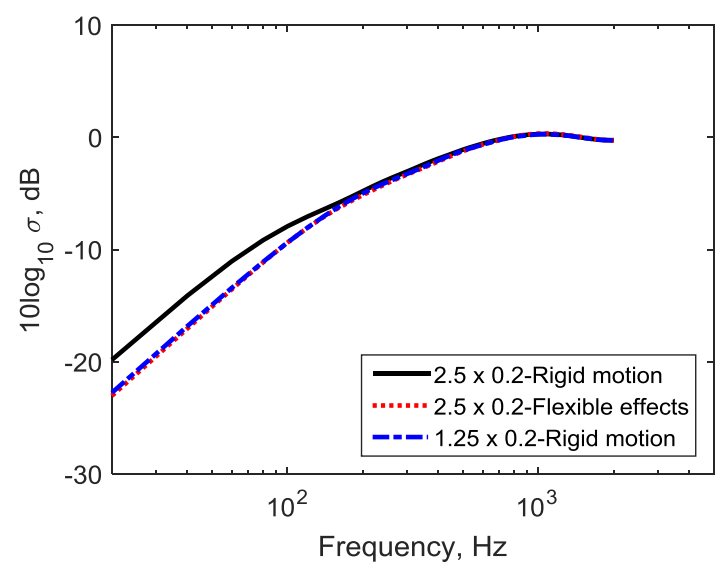

Figure 7. Sound radiation of a single sleeper completely embedded in a rigid ground with an infinite reflection plane

\subsection{Influence of ground absorption on the sleeper radiation}

In practice, the ground is not rigid, but has absorptive properties, which can be described by its acoustic impedance. The absorptive ground surface is introduced in the boundary element model by a finite box, the upper surface of which has the specified impedance. The height of this box is limited to a single element layer and the symmetry plane is introduced beneath it. The ground region close to the sleeper, which is modelled with boundary elements, should be large enough to avoid the effects of diffraction at the edges of the ground box on the sleeper radiation. Three different box models are used for different frequency ranges of interest, as shown in Table 2. The element sizes used here correspond to one quarter of the smallest wavelength in the corresponding frequency range. For the values in the overlapping frequency ranges of 200 $250 \mathrm{~Hz}$ and $800 \sim 1000 \mathrm{~Hz}$ where the boxes give slightly different results (no more than $0.3 \mathrm{~dB}$ ), the results are smoothed by linear interpolation. Again the sleeper is assumed to be completely embedded in the ground with only its upper surface exposed. One quarter of the corresponding model is shown in Figure 8, in which the highlighted region is the upper surface of the sleeper. 
Table 2 Element size and dimensions for the ground box

\begin{tabular}{|c|c|c|}
\hline Frequency range (Hz) & Element size $(\mathrm{m})$ & Box dimensions $(\mathrm{m})$ \\
\hline $20 \sim 250$ & 0.3 & $15 \times 15 \times 0.3$ \\
\hline $200 \sim 1000$ & 0.075 & $4.25 \times 4.25 \times 0.075$ \\
\hline $800 \sim 2000$ & 0.04 & $2 \times 2 \times 0.04$ \\
\hline
\end{tabular}

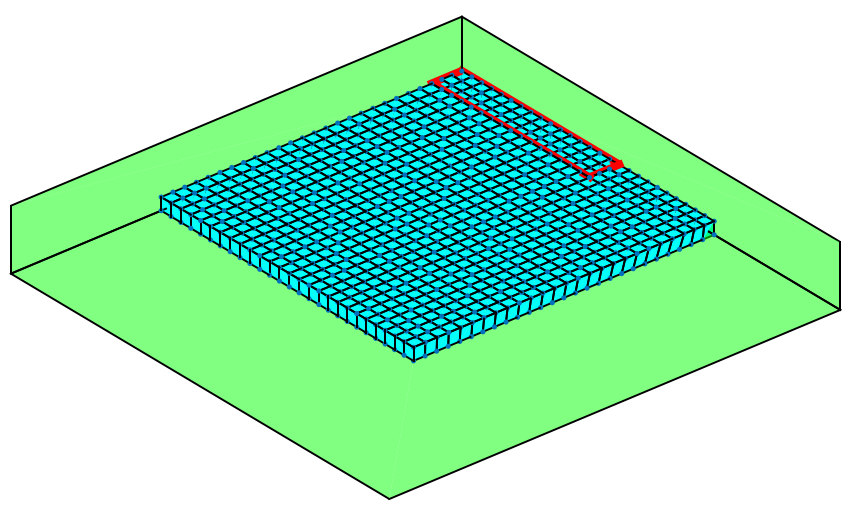

Figure 8. Mesh of one quarter of a single sleeper embedded in absorptive ground at high frequency For simplicity, the Delany and Bazely model [18] is used to determine the ground impedance on the basis of a single parameter, the equivalent flow resistivity. Three values of flow resistivity are used to generate the corresponding impedance for the ground. Figure 9 shows the ground impedances obtained with this model for different values of flow resistivity for an infinitely thick layer of material. As can be seen, the normalised impedance $z_{n} / \rho_{0} c_{0}$ tends to unity as frequency increases, but at low frequency it has large real and (negative) imaginary parts. It can be expected that as the normalised impedance tends to unity, the surface will be able to absorb sound more effectively. As shown in Figure 9, the impedances obtained using the lower values of flow resistivity correspond to impedance values closer to unity and therefore to a more absorptive ground. 



Figure 9. Normalised impedances used for the ground obtained using different values of flow resistivity. $-3 \times 10^{5} ; \cdots 3 \times 10^{4} ;--3 \times 10^{3}$ Pa.s.m ${ }^{-2}$

This simple model for the impedance of the ground surface has been introduced into the boundary element simulations, and the results are compared with those for the rigid ground. Figure 10 shows the radiation ratio. Although the normal velocity is non-zero on the impedance boundary, this is not included in the calculation of the radiation ratio in the denominator of Eq. (1). The result is thus based on sleeper area and velocity. It can be seen that the results for the larger value of flow resistivity, $3 \times 10^{5} \mathrm{~Pa} . \mathrm{s} / \mathrm{m}^{2}$, are quite close to the extreme case of a rigid ground. This means that the sound will mostly be reflected when the impedance is large. For lower values of flow resistivity, the ground is more absorptive, and the sleeper radiates less noise. As can be seen, the radiation ratio is up to $7 \mathrm{~dB}$ lower for the ground with a flow resistivity of $3 \times 10^{3} \mathrm{~Pa} . \mathrm{s} / \mathrm{m}^{2}$. Nevertheless, at very low frequency (below $20 \mathrm{~Hz}$ ) the radiation ratio tends to that of the rigid ground as the corresponding impedance differs from that of air even for this low value of flow resistivity; there is thus a transition between the features of a rigid material and an absorptive material. 


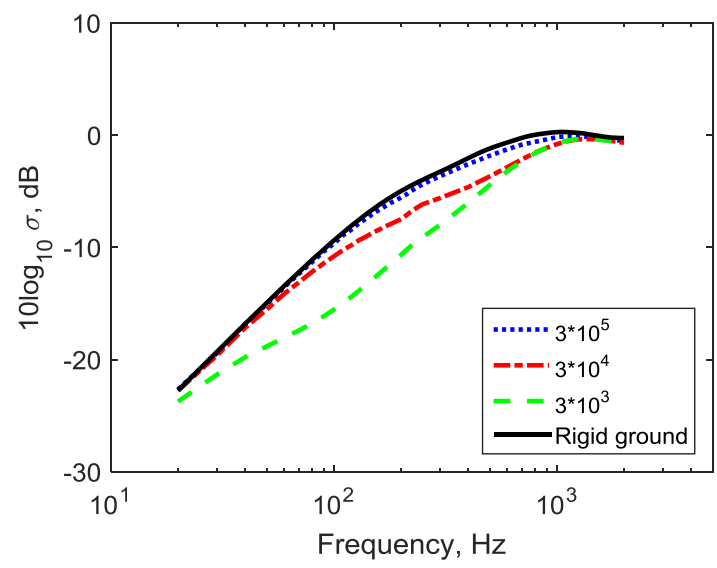

Figure 10. Radiation ratio of single sleeper completely embedded in an absorptive ground with different values of flow resistivity

\section{Sound radiation from multiple sleepers}

\subsection{Vibration of multiple sleepers}

Up to now the radiation from a single sleeper has been considered. In reality, the sleepers are connected to the rails through fasteners and rail pads. When the rail is excited at a point, vibration is transmitted along the rail over a distance that depends on the frequency [10]. At low frequency, due to the track support, wave propagation is inhibited and the rail vibration consists of a near-field region of a certain size. At higher frequency, waves propagate along the rail with a low rate of attenuation; the cut-on frequency of this wave propagation depends on the support stiffness, in particular that of the railpad. The ratio of the sleeper vibration to that of the rail above it is independent of the attenuation of wave propagation in the rail [10]. Thus, if the rail vibrates within a certain distance of the excitation point, all the sleepers within that region also vibrate. The relative amplitude and phase of the various sleepers depends on the corresponding rail vibration. At low frequency, where the acoustic wavelength is large compared with the distance between sleepers, it is expected that multiple sleepers will form a composite source with a modified radiation ratio compared with that for a single sleeper.

To investigate the sound radiation from multiple sleepers, a track vibration model is first used to determine the relative amplitude and phase of the various sleepers. The model used represents the vertical vibration of the track by a continuously supported Timoshenko beam $[10,19]$. Although the discrete sleeper spacing is neglected in this model, the track response is reliably predicted apart from the pinned-pinned frequency region around $1 \mathrm{kHz}$ and the vibration of the sleeper layer at the corresponding distances from the excitation point can be 
taken as representative of the corresponding sleeper vibration amplitudes. The parameters used for the track are listed in Table 3, which represent a track with CEN60E1 rails, concrete monobloc sleepers and relatively soft railpads.

The calculated amplitudes and phases of several sleepers are presented in Figure 11 relative to the vibration of sleeper 1, which is directly beneath the excitation point. As can be seen, below about $300 \mathrm{~Hz}$ the vibration amplitude of sleeper 2, at $0.6 \mathrm{~m}$ away from the excitation point, can be approximated as being half that of sleeper 1 at $0 \mathrm{~m}$, and they are roughly in phase. Subsequent sleepers have a much smaller vibration amplitude in this low frequency region, which is characterised by near-field behaviour in the rail. The vibration ratios become closer to unity and obvious phase changes occur above about $400 \mathrm{~Hz}$, which is the region in which bending waves propagate in the rail. For a track with stiffer railpads, not shown, the low frequency near-field behaviour of the rail is similar to this but extends up to a higher frequency.

Table 3 Parameters used for vertical motion of the track in the calculations

\begin{tabular}{|l|l|}
\hline Rail bending stiffness & $6.42 \mathrm{MNm}^{2}$ \\
\hline Rail mass per unit length & $60 \mathrm{~kg} / \mathrm{m}$ \\
\hline Rail shear stiffness & $6.17 \times 10^{8} \mathrm{~N}$ \\
\hline Rail shear parameter & 0.4 \\
\hline Rail rotational inertia & $0.24 \mathrm{~kg} \cdot \mathrm{m}$ \\
\hline Pad stiffness & $120 \mathrm{MN} / \mathrm{m}$ \\
\hline Pad damping loss factor & 0.2 \\
\hline Sleeper mass (half) & $150 \mathrm{~kg}$ \\
\hline Sleeper spacing & $0.6 \mathrm{~m}$ \\
\hline Ballast stiffness & $100 \mathrm{MN} / \mathrm{m}$ \\
\hline Ballast loss factor & 1.0 \\
\hline
\end{tabular}



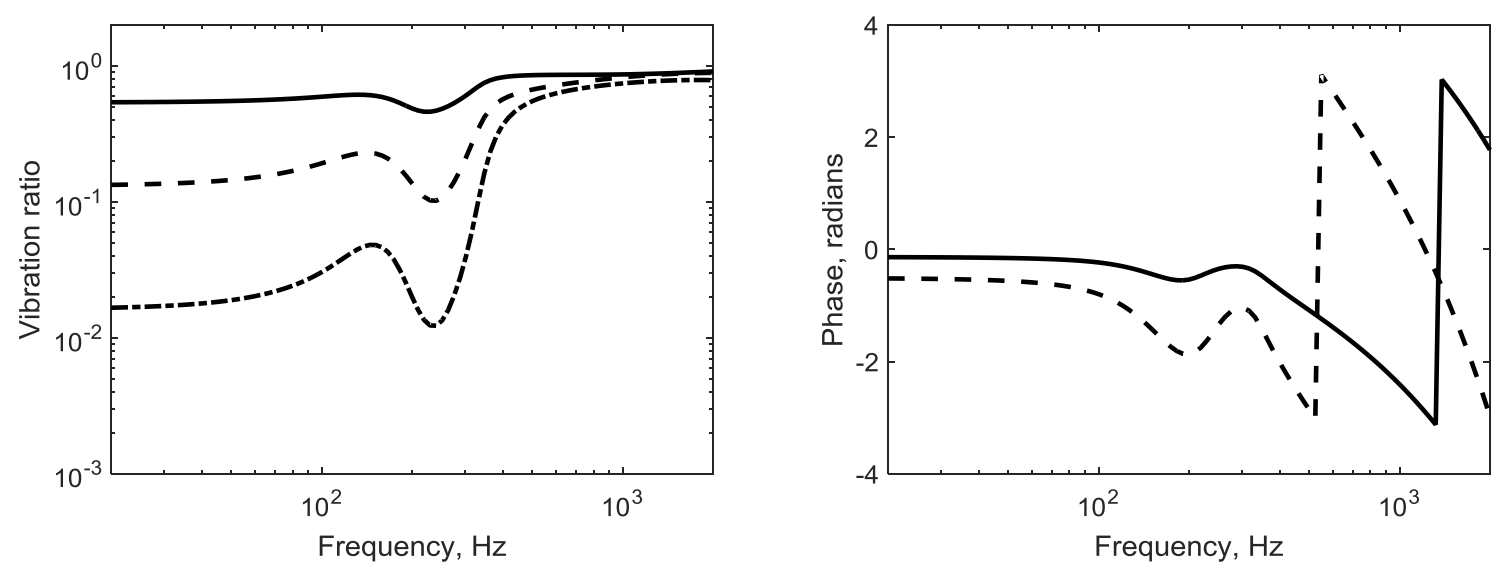

Figure 11. Vibration of successive sleepers relative to sleeper 1 at $0 \mathrm{~m} .-$, sleeper 2 at $0.6 \mathrm{~m}$; - - sleeper 3 at $1.2 \mathrm{~m} ;-\cdot-\cdot$, sleeper 6 at $3.0 \mathrm{~m}$ (amplitude only)

\subsection{Sound radiation of multiple sleepers in rigid ground}

The sound radiation from multiple sleepers is calculated first when the sleepers are embedded in a rigid ground. For convenience the Rayleigh integral method has been used as this more readily allows larger models to be used. In each case the upper surface of the sleepers is assumed to be flush with the rigid ground. As before, the sleepers in the radiation model have a length of $1.25 \mathrm{~m}$ and are assigned rigid vertical motion.

The sound radiation obtained for three sleepers is shown in Figure 12. The outer sleepers are assigned a vibration amplitude of half that of the central one and all are assumed to be vibrating in phase; this corresponds to the low frequency behaviour seen in Figure 11. To show the influence of the vibration ratio, results are also shown for three sleepers with equal vibration amplitude; these are very similar to the results obtained for a vibration ratio of 0.5 with a difference of no more than $0.6 \mathrm{~dB}$. However, if the vibration ratio is 0.2 for example, the low frequency results would be reduced by around $2 \mathrm{~dB}$. For comparison the radiation ratio of an individual sleeper in a rigid baffle is also shown (from Figure 6). As can be seen, all the result tends to unity at high frequencies. At low frequencies, however, the radiation ratio of the three sleepers is higher than that of the single sleeper by up to $5 \mathrm{~dB}$, indicating that they will radiate more noise than if they were considered separately. The acoustic wavelength is equal to $1.2 \mathrm{~m}$, twice the sleeper separation distance, at $286 \mathrm{~Hz}$, above which the radiation ratio drops. The dip at around $400 \mathrm{~Hz}$ occurs due to interference between the radiation of the individual sleepers. A peak occurs at $570 \mathrm{~Hz}$, where the acoustic wavelength equals $0.6 \mathrm{~m}$, the sleeper separation distance. 


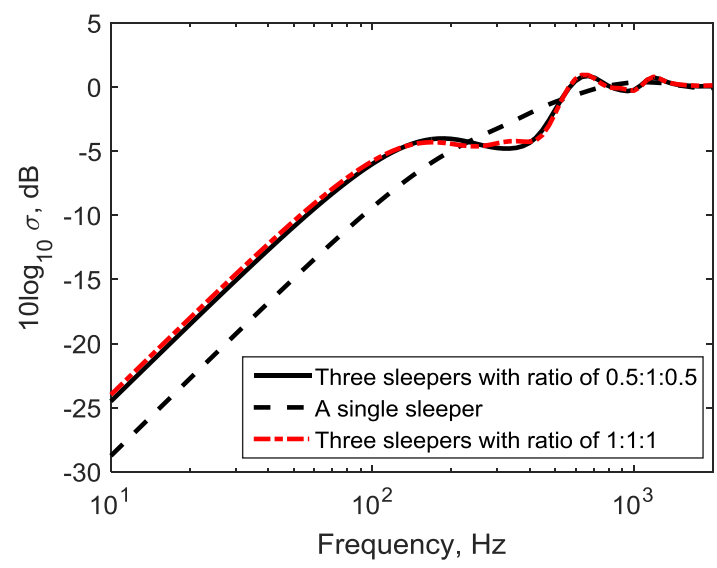

Figure 12. Radiation ratio of sleepers set in a rigid baffle

Figure 13 shows the increase in radiation ratio that occurs when multiple sleepers are considered relative to the result for a single sleeper. In this figure the effect of vibration decay and propagation along the rail is neglected; it will be considered explicitly below. Here, results are shown for three, five and nine sleepers, each with identical vibration amplitudes. It can be seen that the results rise to a constant value at low frequency. For three sleepers this is an increase in radiation ratio of $5 \mathrm{~dB}$ for frequencies below about $100 \mathrm{~Hz}$. As the number of sleepers increases, the size of the composite source becomes greater. Consequently, a higher value of radiation ratio is reached at the low frequency asymptote, but this value is not reached until a lower frequency than for the case of less sleepers. In the region between $100 \mathrm{~Hz}$ and $250 \mathrm{~Hz}$ the radiation ratio tends to that of the single sleeper and is independent of the number of sleepers included in the model. The acoustic wavelength is equal to twice the sleeper spacing $(1.2 \mathrm{~m})$ at $285 \mathrm{~Hz}$. Above this frequency the results oscillate within the region $\pm 2 \mathrm{~dB}$, tending to $0 \mathrm{~dB}$ at high frequency (above $1.5 \mathrm{kHz}$ ).

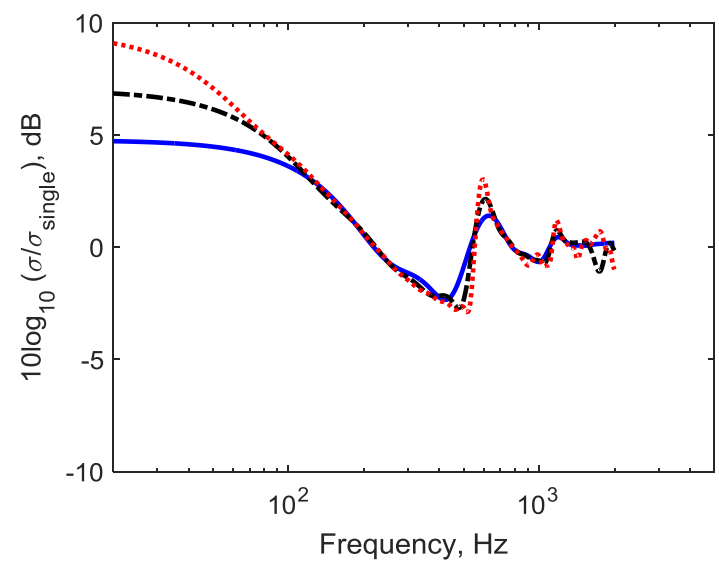

Figure 13. Increase in sleeper radiation ratio due to multiple sleepers:,- 3 sleepers with vibration amplitudes in the ratio $1: 1: 1 ;-\cdot-\cdot, 5$ sleepers; $\cdots, 9$ sleepers 
Figure 14 shows corresponding results for three sleepers with a vibration ratio of 0.5 between the outer and central sleeper, as used in Figure 12. This is compared with results in which the vibration amplitudes are obtained from the track vibration model, i.e. using the results from Figure 11. The increase in the radiation ratio calculated in this way is shown for three sleepers and 15 sleepers. Similar results are found in each of these three cases, especially at low frequency (below $400 \mathrm{~Hz}$ ). Below $100 \mathrm{~Hz}$ the increase in radiation ratio is approximately constant at $4.5 \mathrm{~dB}$. This increase is now virtually independent of the number of sleepers included in the model due to the fact that the sleepers further from the excitation point on the rail have much lower amplitudes. Between 100 and $250 \mathrm{~Hz}$ the radiation ratio again tends to that of the single sleeper; above this frequency the results again oscillate within the region \pm 2 $\mathrm{dB}$.

From these results it can be concluded that it is sufficient to consider only three sleepers in determining the radiation ratio for predicting the noise from the whole track system. Although more sleepers are contained within the acoustic wavelength at low frequency, the rail vibration is localised so that those additional sources have negligible source strength. At high frequencies, although the rail decay rate drops and many sleepers vibrate together, the acoustic wavelength is shorter and they can be considered independent sources. Therefore, in the remainder of this paper, work is focussed on the use of three sleepers to represent multiple sleepers in the track.

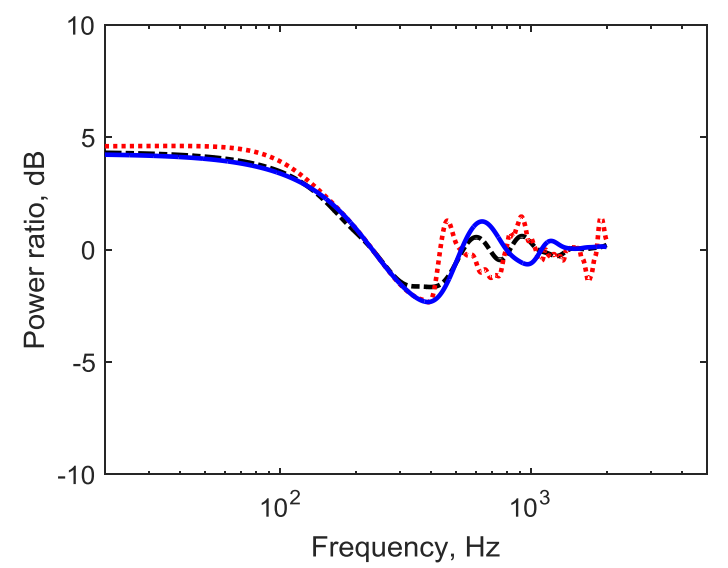

Figure 14. Increase in sleeper radiation ratio due to multiple sleepers: - , three sleepers with vibration amplitudes in the ratio $0.5: 1: 0.5 ;-\cdot-\cdot$, three sleepers with amplitudes from track vibration model; $\cdots$, 15 sleepers with amplitudes from track vibration model

\subsection{Multiple sleepers in absorptive ground}

The sound radiation for three sleepers in proximity to an absorptive ground is explored here using the boundary element model. As above, the outer sleepers are assigned a vibration 
amplitude of half that of the central sleeper; all three are assumed to be vibrating in phase. As in Section 2.4, the dimensions of the box representing the ground were determined first using a rigid ground box. The element size and box dimensions of the ground in different frequency ranges are taken to be the same as in Table 2 .

The sound radiation of the sleepers vibrating vertically when they are embedded in an absorptive ground is obtained for a ground with various values of flow resistivity. Figure 15 shows the results. As for a single sleeper (Figure 10), it can be seen that the ground will absorb more noise if it has a lower flow resistivity. For example, the sound radiation will be reduced by up to $5 \mathrm{~dB}$ for the ground with flow resistivity of $3 \times 10^{3} \mathrm{~Pa} . \mathrm{s} / \mathrm{m}^{2}$, compared with a rigid ground. However, the reduction will be much smaller at high frequency and is negligible above $1 \mathrm{kHz}$. In the same way as for the single sleeper, at very low frequencies the results tend towards those for a rigid ground as the impedance increases.

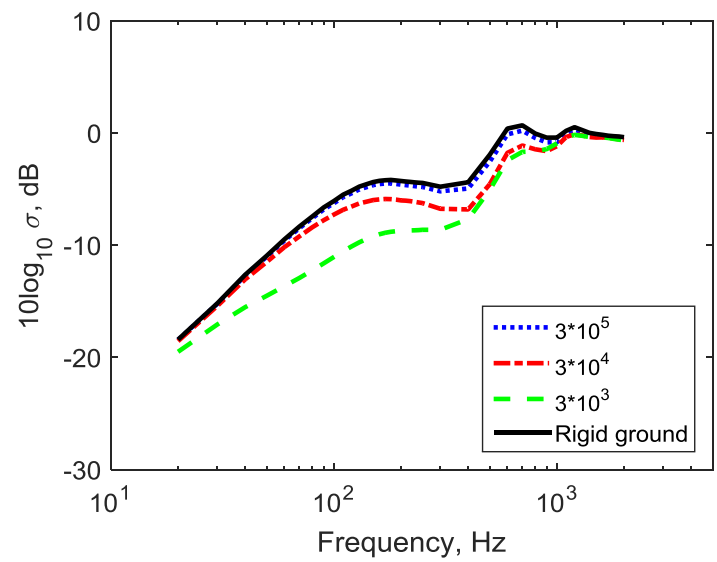

Figure 15. Radiation ratio of multiple sleepers completely embedded in an absorptive ground with different flow resistivity

\section{Experimental validation}

Measurements have been carried out to investigate the sound radiation of a single sleeper and three connected sleepers by using a reciprocal method [20]. Scale model sleepers, as shown in Figure 16, are used. They have dimensions corresponding to $1 / 5$ of the sleeper considered in the previous sections and were cast from concrete. The sleeper radiation has been determined experimentally in different configurations for vertical vibration. In each configuration two sets of measurements are required to determine the radiation ratio (see Eq. (1)): the transfer mobility to give the average mean-square velocity and the sound power normalised to a unit mean square force. To determine the spatially averaged mobility, a miniature impact hammer with a titanium 
tip has been used to excite the sleeper, which provides a sufficient excitation up to $10 \mathrm{kHz}$. A reciprocal method [20] has been used to obtain the acoustic transfer function of the sleeper (power for a unit mean square force) by placing the sleeper in a reverberation chamber and measuring its response to acoustic excitation. Further details of this method are given in [20].

Measurements have been carried out for a single sleeper in free space, a sleeper embedded in melamine foam to represent an absorptive ground and finally for three sleepers connected by a short length of rail and resting on the foam. In each case comparisons are made between the measured results and numerical predictions carried out for the 1/5 scale model.

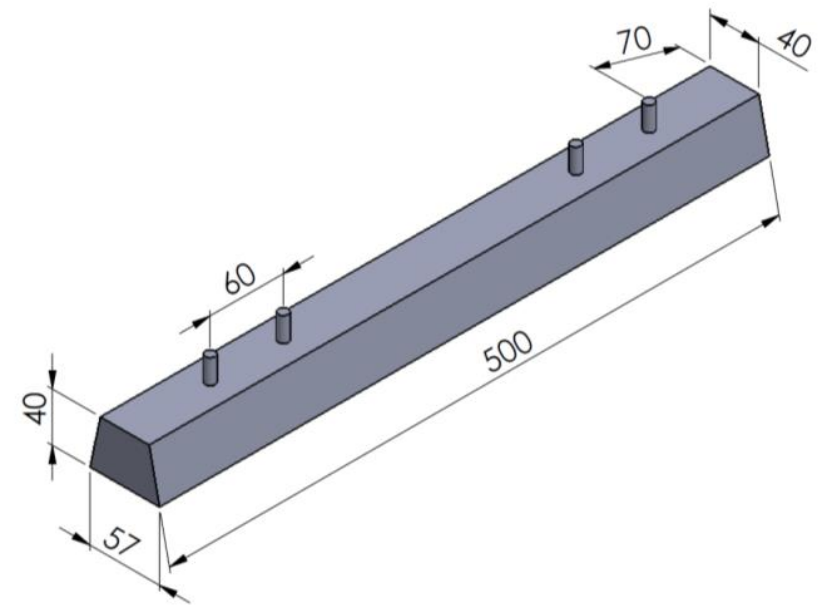

Figure 16. Scale model sleeper, dimensions in $\mathrm{mm}$

\subsection{A single sleeper in free space}

The sound radiation of a single sleeper has been measured first with the sleeper in free space. Structural reciprocity has been used during the mobility tests, so that the hammer is moved along the length of the sleeper and the response is measured at the three fixed 'excitation' positions. For the spatially averaged mobility, 11 excitation points were used, spaced equally along the sleeper, and one accelerometer position, located at $0.1 \mathrm{~m}$ from one end of the sleeper; this corresponds to the rail seat. For each force position, the frequency response function was obtained as an average over 5 impacts.

The acceleration response of the structure to a measured sound pressure field has been obtained in a reverberation chamber of volume $348 \mathrm{~m}^{3}$. Four loudspeakers, excited by white noise over the frequency range up to $10 \mathrm{kHz}$, were used to create the sound field. The spatially averaged sound pressure in the room has been obtained approximately using a microphone placed on a rotating boom. 
To simulate a sleeper in free space, the scale model sleeper was suspended $1 \mathrm{~m}$ above the floor of the reverberation chamber and the room was excited by an acoustic source. A comparison of the measured sound pressure level with the background noise in the chamber is shown in Figure 17(a). Figure 17(b) presents equivalent results for the vertical acceleration signal on the sleeper. As can be seen, an adequate signal-to-noise ratio is achieved for both the sound pressure and the acceleration between about $160 \mathrm{~Hz}$ and $7 \mathrm{kHz}$. Figure 18(a) presents the normalised power obtained by the reciprocity method. The spatially averaged transfer mobility of the sleeper is shown in Figure 18(b). These results are all shown in one-twelfth octave bands. This provides some smoothing of the measured data, while retaining a finer resolution than the more usual one-third octaves. Peaks can be seen in the normalised power and average mobility at the natural frequencies of the $1 / 5$ scale sleeper: $550 \mathrm{~Hz}, 1500 \mathrm{~Hz}, 2800 \mathrm{~Hz}, 4400 \mathrm{~Hz}$, etc.

(a)

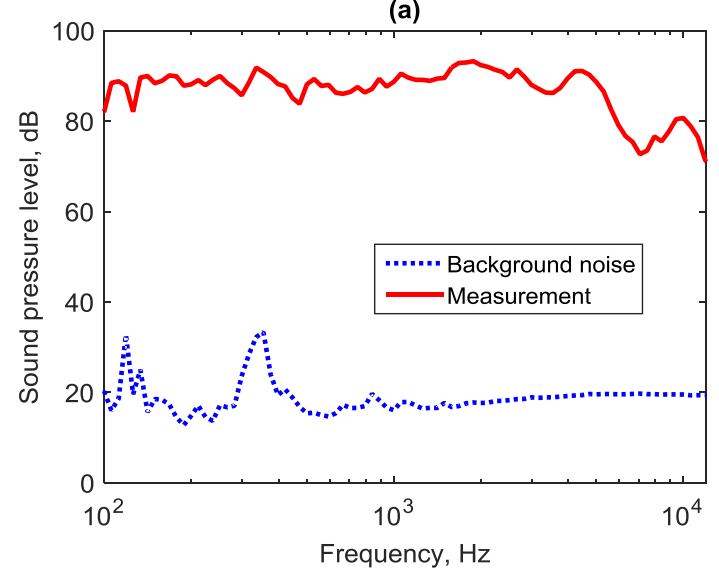

(b)

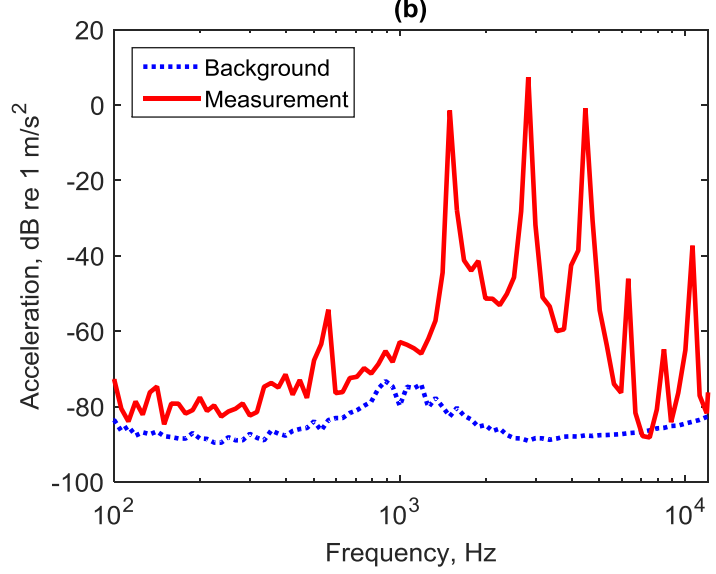

Figure 17. Comparison of measured spectra and background noise signals in one-twelfth octave bands for sleeper in reverberation chamber. (a) Sound pressure level; (b) acceleration
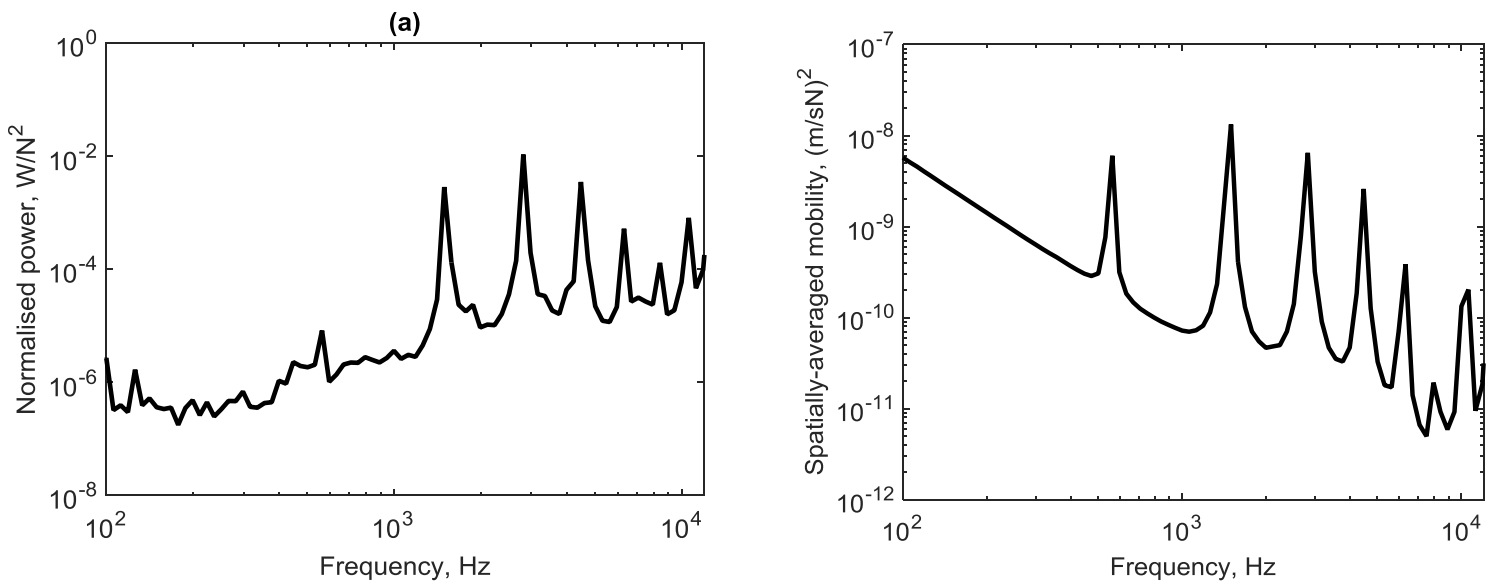

Figure 18. (a) Normalised sound power and (b) spatially-averaged mobility of sleeper in onetwelfth octave bands for single sleeper in free space 
Figure 19 presents the radiation ratio of the sleeper obtained from these results. It can be seen that the experimental results agree well with the numerical prediction from a 3D BEM model of the sleeper, especially at high frequency (between $1.5 \mathrm{kHz}$ and $6 \mathrm{kHz}$ ). At low frequency (below $1.5 \mathrm{kHz}$ ), the sleeper sound radiation is overestimated, especially at around $550 \mathrm{~Hz}$. This is because, in this initial BEM model, the sleeper is assumed to vibrate with rigid body motion. In practice, however, the sleeper has bending modes, as seen in Figure 18. The dip in the radiation ratio at around $550 \mathrm{~Hz}$ corresponds to the first bending mode of the sleeper. Above $5 \mathrm{kHz}$, where the radiation ratio should tend to unity, the measured results appear too high. The reason for this is unclear.

The effects of the sleeper flexibility have therefore been taken into account in the numerical model. The vibration is calculated using the free-free finite Timoshenko beam model as in Section 2.1. Its sound radiation is determined using the three-dimensional boundary element model with this vibration as input. These results are also presented in Figure 19. It can be seen that they agree very well with the measurements, with the dip in the measurements at around $550 \mathrm{~Hz}$ reproduced in the numerical predictions.

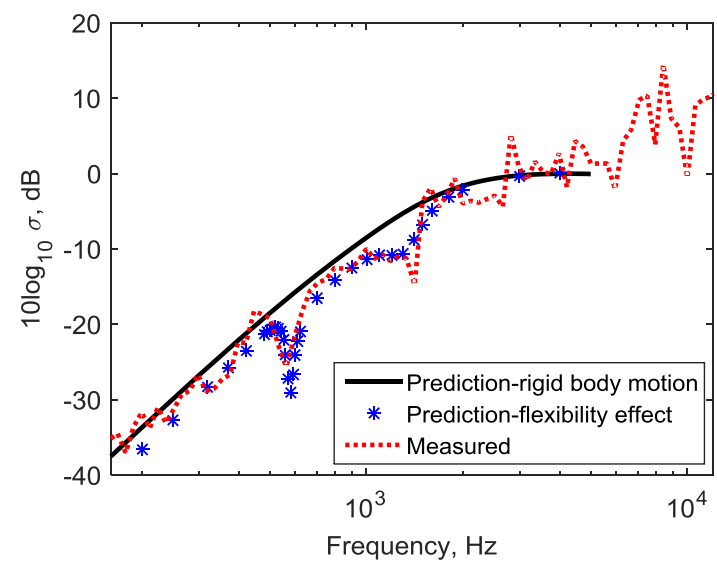

Figure 19. Comparison of radiation ratio from numerical prediction and measurement for the scale model sleeper in free space

\subsection{A single sleeper embedded in foam}

The effect of an absorptive ground on the sleeper radiation has been tested by embedding the sleeper in melamine foam, as shown in Figure 20. For this purpose, pieces of foam were cut to shape to match the profile of the sleeper. The overall dimensions of the foam were $1 \mathrm{~m} \times 0.4$ $\mathrm{m} \times 0.1 \mathrm{~m}$. 


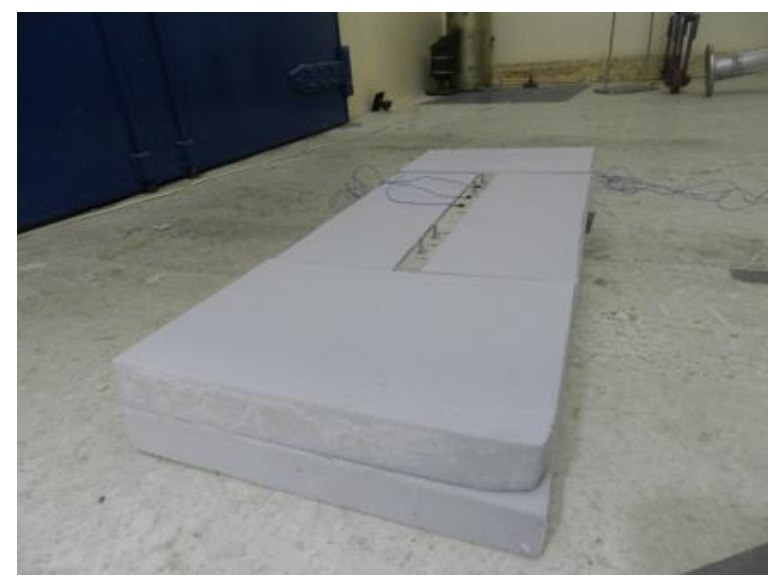

Figure 20. Sleeper embedded in melamine foam

An equivalent value of the flow resistivity has been deduced as $8000 \mathrm{~Pa} . \mathrm{s} / \mathrm{m}^{2}$ by fitting the Delany-Bazley model [18] to the results of impedance tube measurements. This value is used in the numerical calculation. Figure 21 presents the measurement results and the corresponding numerical predictions. Good agreement can be seen between them. At low frequency (below $400 \mathrm{~Hz}$ ), the numerical results are slightly higher than the measurements. Above $5 \mathrm{kHz}$, the measured results again appear too high.

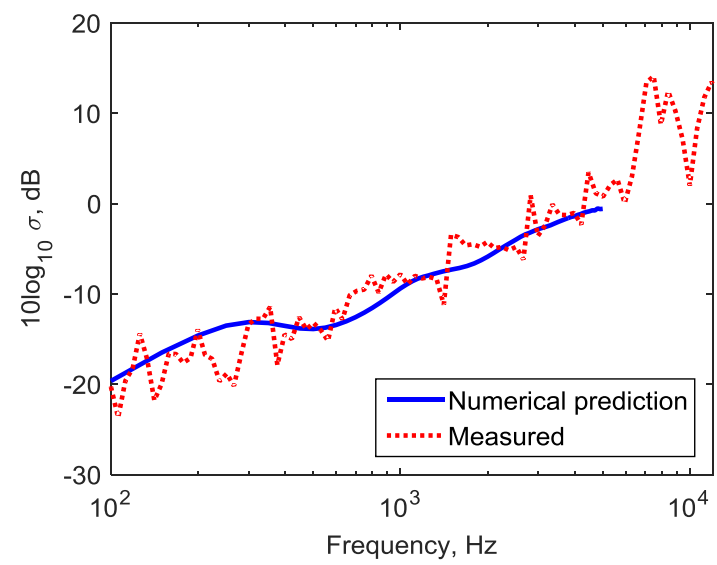

Figure 21. Comparison of measured radiation ratio and numerical predictions for a single sleeper embedded in melamine foam

\subsection{Multiple sleepers}

The sound radiation from three sleepers has also been investigated experimentally. Three sleepers were connected by two short $1 / 5$ scale rails of length $0.36 \mathrm{~m}$. These were attached to the sleepers using spring clips; there were no rail pads between the rails and the sleepers. The sleepers rested on melamine foam with dimensions $1.2 \mathrm{~m} \times 1 \mathrm{~m} \times 0.05 \mathrm{~m}$, as shown in Figure 22. The assembly was measured in the reverberation chamber using the same method as previously. The transfer mobility was measured for excitation at 13 points spaced equally along 
each rail and 11 points spaced equally along each sleeper, with two accelerometers located at the centre of the railhead above the central sleeper on each rail. By reciprocity these measurements represent the transfer mobility from a force at the railhead to the velocity on the rail or sleeper.

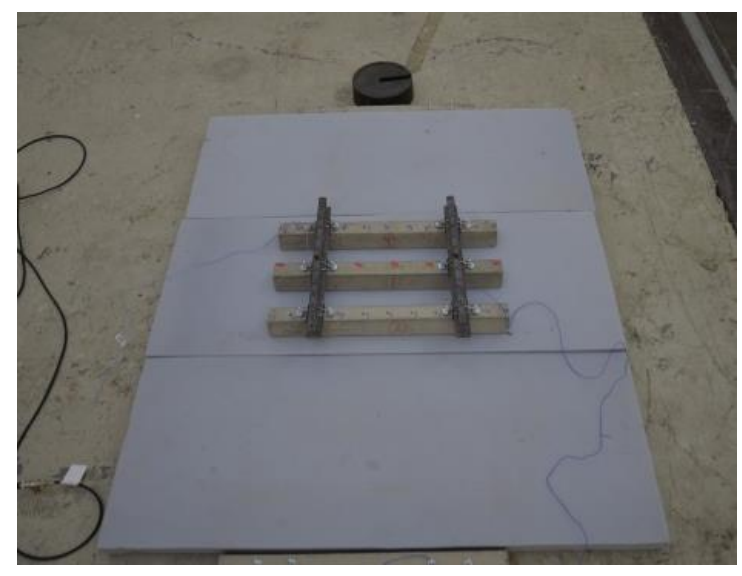

Figure 22. Experimental set-up for three sleepers resting on foam

Figure 23 shows the ratio of the averaged mobility amplitude of the outer sleepers to that of the middle one. As can be seen, this mobility ratio differs from those shown in Figure 11, mainly because of the short length of the rails in the measurement.

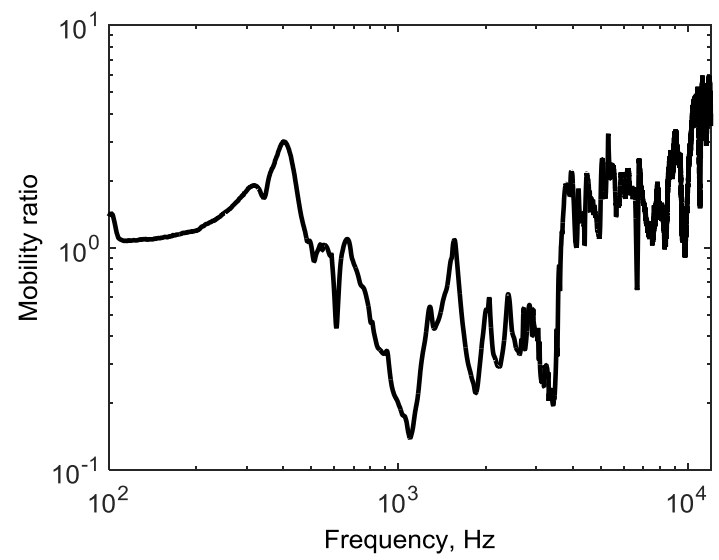

Figure 23. Averaged mobility amplitude of the outer sleepers relative to the central one

Figure 24 presents the measured sound power from the three sleepers and two short rails normalised by the mean square velocity of the central sleeper. For comparison the sound power from the rails has been estimated by using the corresponding radiation ratio from [13]. For this a weighted average of the radiation ratio of a rail attached to a rigid ground and a rail above an absorptive ground is used. These results are again shown normalised by the mean square velocity of the central sleeper. Although this is only a rough estimate, it shows that the sound power from the rail gives the dominant contribution at high frequency, especially above 2.2 
$\mathrm{kHz}$ (which corresponds to $440 \mathrm{~Hz}$ for the full size track system), whereas the sleeper radiation dominates at low frequency. Thus it may be concluded that the contribution from the rails can be ignored below $2 \mathrm{kHz}$ in the present experiment.



Figure 24. Measured sound power for three sleepers connected by rails resting on melamine foam. The power is shown normalised by the mean square velocity of the central sleeper. For comparison the estimated sound power from the rails is also shown

Finally, the sound power from three connected sleepers is compared with the power that would be radiated by three single sleepers. Numerical and experimental results are shown in Figure 25 in the form of the ratio between the power from the connected sleepers and that from the same sleepers radiating individually. In the predictions the vibration of the outer sleepers is assigned an amplitude equal to the measured mobility ratio from Figure 23 while the central sleeper has unit vibration amplitude. Measured results are not shown below $160 \mathrm{~Hz}$, where the signal-to-noise ratio in the sound power measurement was very low. As can be seen, reasonable agreement is found between the numerical and measured results, which both show a similar tendency to the earlier predictions in Figure 14. Above $2 \mathrm{kHz}$ the measured results are higher than the predictions, due to the dominant contribution of the rails. 


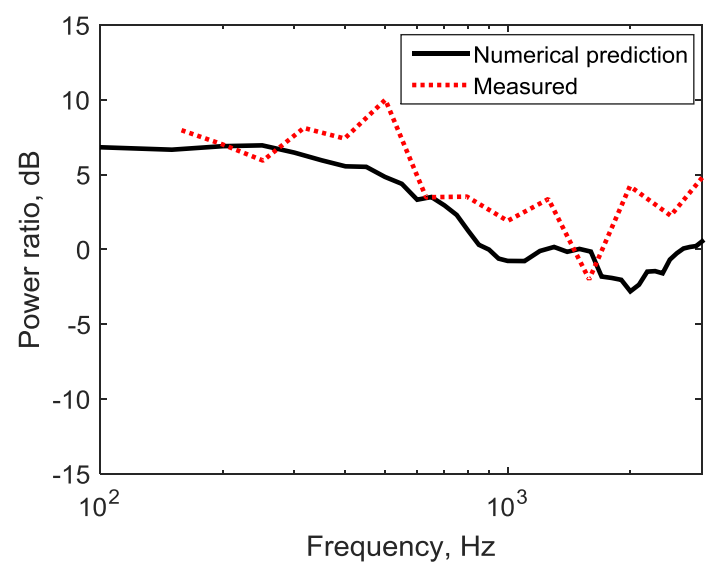

Figure 25. Ratio of sound power radiated by three connected sleepers to that obtained for the three sleepers separately

\section{Conclusions}

The acoustic characteristics of railway sleepers undergoing vertical vibration have been predicted using a three-dimensional boundary element model. It has been shown that a single sleeper in free space exhibits the behaviour of a point dipole at low frequencies, but follows the behaviour of a line dipole in intermediate frequencies before tending to unity. When the sleeper is embedded in a rigid ground the corresponding behaviour is that of a point monopole at low frequencies and a line monopole at intermediate frequencies. When the sleeper flexibility and support stiffness are taken into account, the radiation ratio of a sleeper of length $2.5 \mathrm{~m}$ can be approximated by that for a rigid half-sleeper of length $1.25 \mathrm{~m}$. Furthermore the effect of ground absorption has been taken into account and shown to reduce the radiation from the vertical motion of the sleeper especially in the mid-frequency region.

When multiple sleepers are excited through the rail, their sound radiation is greater than would be obtained from the same sleepers treated independently. This increase in sound radiation has been calculated for cases where the sleeper is embedded in a rigid or partially absorptive ground. It is shown that it is sufficient to consider only three sleepers in determining their radiation ratio when installed in track. At low frequencies, although more sleepers lie within an acoustic wavelength, the vibration of the track is localised to the three sleepers nearest the excitation point. At higher frequencies where more sleepers have significant vibration the distance between them is large enough for them to be treated independently. Consequently the sound radiation increases by up to $5 \mathrm{~dB}$ below $100 \mathrm{~Hz}$ whereas above $300 \mathrm{~Hz}$ the result can be approximated by that for a single sleeper. 
Measurements have been carried out on a 1/5 scale model railway track to verify the numerical predictions for various configurations. Good agreement has been found between the measured results and the numerical predictions for all configurations.

\section{Acknowledgements}

The work described has been supported by the EPSRC under the programme grant EP/H044949/1, 'Railway Track for the $21^{\text {st }}$ Century' (Track 21).

\section{References}

[1] Remington, P.J., Wheel/rail noise, part IV: rolling noise, Journal of Sound and Vibration, 46, 419-436, (1976).

[2] Remington, P.J., Wheel/rail rolling noise, I: Theoretical analysis, Journal of the Acoustical Society of America, 81, 1805-1823, (1987).

[3] D.J. Thompson, B. Hemsworth and N. Vincent. Experimental validation of the TWINS prediction program for rolling noise, part 1: description of the model and method. Journal of Sound and Vibration, 193, 123-135, 1996.

[4] D.J. Thompson, P. Fodiman and H. Mahé. Experimental validation of the TWINS prediction program, part 2: results. Journal of Sound and Vibration, 193, 137-147, 1996.

[5] C.J.C. Jones, D.J. Thompson, Extended validation of a theoretical model for railway rolling noise using novel wheel and track designs. Journal of Sound and Vibration 267, 509-522, 2003.

[6] Track Wheel Interaction Noise Software (TWINS) User manual. 1996.

[7] N. Vincent, P. Bouvet, D. J. Thompson and P. E. Gautier. Theoretical optimisation of track components to reduce rolling noise. Journal of Sound and Vibration. 193, 161-171, 1996.

[8] J.C.O. Nielsen. Acoustic optimization of railway sleepers. Journal of Sound and Vibration, 231, 753-764, 2000.

[9] B. Hemsworth, P. E. Gautier and R. Jones. Silent Freight and Silent Track projects. Proceedings of Internoise 2000, Nice, France 2000.

[10] D. Thompson. Railway noise and vibration mechanisms, modelling and means of control. Elsevier, Oxford, 2008.

[11] J.W. Strutt (Lord Rayleigh). Theory of Sound, $2^{\text {nd }}$ edition. Dover Publications, New York, 1945. 
[12] K. Attenborough, P. Boulanger, Q. Qin, and R. Jones. Predicted influence of ballast and porous concrete on rail noise. Internoise, Brazil, 2005.

[13] X. Zhang, D.J. Thompson and G. Squicciarini. Influence of ground impedance on the sound radiation of a railway track. In: Proceedings of $21^{\text {st }}$ International Congress on Sound and Vibration, Beijing, China, 2014.

[14] K. Knothe, S.L. Grassie. Modelling of railway track and vehicle/track interaction at high frequencies. Vehicle System Dynamics, 22, 209-262, 1993.

[15] S.L. Grassie. Dynamic modelling of concrete railway sleepers. Journal of Sound and Vibration. 187, 799-813, 1995.

[16] F. Fahy, P. Gardonio. Sound and structural vibration: radiation, transmission and response. Second edition, Elsevier, Oxford, 2007.

[17] T.W. Wu. Boundary element acoustics: Fundamentals and computer codes. WITpress, 2000 .

[18] M.E. Delany, E.N. Bazley. Acoustical properties of fibrous absorbent materials. Applied Acoustics, 3, 105-116, 1970.

[19] S.L. Grassie, R.W. Gregory, D. Harrison, and K.L. Johnson. The dynamic response of railway track to high frequency vertical excitation. Journal of Mechanical Engineering Science, 24, 77-90, 1982.

[20] G. Squicciarini, A. Putra, D.J. Thompson, X. Zhang and M.A. Salim. Use of a reciprocity technique to measure the radiation efficiency of a vibrating structure. Applied Acoustics, 89, 107-121, 2015. 